\title{
Determinação das propriedades geométricas, físicas e mecânicas do sistema fruto-pedúnculo-ramo do cafeeiro
}

\author{
Andre L. de F. Coelho ${ }^{1}$, Fábio L. Santos ${ }^{2}$, Francisco A. C. Pinto ${ }^{3} \&$ Daniel M. de Queiroz ${ }^{4}$
}

\section{Palavras-chave: \\ Catuaí Vermelho \\ coeficiente de Poisson \\ massa específica \\ módulo de elasticidade \\ razão de amortecimento}

\begin{abstract}
R E S U M O
O método de elementos finitos tem sido empregado no projeto de máquinas e para o estudo do comportamento dinâmico do cafeeiro. Objetivou-se, neste trabalho, determinar as propriedades geométricas, físicas e mecânicas do sistema fruto-pedúnculo-ramo do cafeeiro. As dimensões foram determinadas por meio de processamento de imagens, as massas e os volumes mensurados utilizando-se balança e proveta, respectivamente. Os módulos de elasticidade foram determinados por meio de ensaios mecânicos de compressão, tração e flexão, para os frutos, pedúnculos e ramos, respectivamente. Os coeficientes de Poisson foram determinados a partir das deformações longitudinais e transversais dos frutos e pedúnculos. A razão de amortecimento foi determinada por meio das curvas de decaimento utilizando-se o método de decremento logaritmo. Concluiu-se que as dimensões e a massa dos frutos apresentaram tendência de aumento na medida em que seu estádio de maturação evoluiu. Os módulos de elasticidade dos frutos e dos pedúnculos no estádio de maturação verde foram superiores aos valores para o estádio de maturação cereja.
\end{abstract}

Key words:

Red Catuai

Poisson's ratio

density

elasticity modulus

damping ratio

\section{Determination of geometric, physical and mechanical properties of coffee fruit-stem-branch system}

\begin{abstract}
A B S T R A C T
The finite element method has been employed in the machine design, as well as to study the dynamic behavior of the coffee plant or its parts. The objective of this study was to determine the geometric, physical and mechanical properties of coffee fruit-stem-branch system. The dimensions were determined by image processing, the masses and volumes measured using weighing-machine and graduated cylinder, respectively. The modulus of elasticity was determined by compression, tension and bending mechanical tests for fruits, stems and branches, respectively. The Poisson's ratio was determined using the longitudinal and transversal deformation of the fruits and stems. The damping ratio was determined by the decay curves using the logarithmic decrement method. It was concluded that the fruit dimensions and mass showed a tendency to increase with mature stage evolution. The modulus of elasticity for fruit and stems in green (cherry) stage was higher than the values for the mature stage.
\end{abstract}




\section{INTRODUÇÃo}

O café é um contribuinte importante para o produto interno bruto (PIB) do Brasil. Como maior produtor mundial, sua produção foi, em 2012, de 50,83 milhões de sacas de 60 quilos. Para 2013 a estimativa de produção foi de 48,59 milhões de sacas cuja previsão de redução se deve principalmente ao ciclo de baixa bienalidade e à irregularidade das chuvas associadas a altas temperaturas em grande parte das áreas de café arábica (CONAB, 2013).

A colheita do café nos sistemas manuais e semimecanizados é realizada em seis etapas: arruação, derriça, varrição, recolhimento, abanação e transporte. A derriça é uma etapa que demanda elevado contingente de mão de obra contribuindo significativamente para o custo de colheita (Barbosa et al., 2005). Assim, o desenvolvimento de máquinas para a derriça dos frutos se torna imprescindível para aumentar a capacidade operacional do processo de colheita e, por sua vez, amenizar os problemas relativos à escassez de mão de obra no setor agrícola.

Atualmente, as máquinas empregadas para a colheita mecanizada de culturas, como café, damasco, laranja, pistache, oliva e uva, utilizam o princípio de vibrações mecânicas (Erdogan et al., 2003; Sanders, 2005; Sessiz \& Ozcan, 2006; Souza et al., 2006; Polat et al., 2007; Pezzi \& Caprava, 2009; Santos et al., 2010a). Por meio de fontes de potência elétrica, pneumática, hidráulica ou mecânica, a energia cinética é transmitida à planta ou a uma parte dela, promovendo o desprendimento dos frutos.

A colheita mecanizada por vibrações mecânicas exige uma combinação ótima dos parâmetros frequência, amplitude e tempo de vibração, o que permitirá maior eficiência no desprendimento dos frutos além de promover uma desfolha menor e quebra de ramos. A determinação desses parâmetros pode ser realizada a partir do estudo do comportamento dinâmico da planta a ser colhida (Ciro, 2001; Santos et al., 2010a).

O comportamento dinâmico da planta de café, ou de parte dela, vem sendo estudado por meio de equações analíticas, experimentos controlados em laboratório, experimentos de campo ou ferramentas computacionais (Ciro, 2001; Filgueiras, 2001; Aristizábal et al., 2003; Souza et al., 2006; Santos et al., 2010a; 2010b,). Esta última compreende o método de elementos finitos que consiste na geração e na solução de um sistema de equações diferenciais que governam o comportamento físico em questão (Alburqueque, 2005; Taplak \& Parlak, 2012). No entanto, para a realização de simulações computacionais que permitirão o estudo de diferentes cenários, são demandados parâmetros de entrada referentes às propriedades geométricas, físicas e mecânicas do sistema.

Ao estudar a variedade Colombiana do cafeeiro, Ciro (2001) determinou as propriedades físicas, geométricas e mecânicas do sistema fruto-pedúnculo em três estádios de maturação; posteriormente, o autor realizou a modelagem do sistema fruto-pedúnculo como um sistema de um e dois graus de liberdade determinando, analiticamente, as frequências naturais de vibração e concluiu que as frequências naturais do sistema fruto-pedúnculo são dependentes das propriedades físicas e mecânicas. O autor verificou, também, que tais frequências reduzem na medida em que o estádio de maturação do sistema fruto-pedúnculo evolui de verde para cereja.

Com o objetivo de avaliar as propriedades mecânicas dos frutos de café, Couto et al. (2002) determinaram o módulo de deformidade de frutos do cafeeiro para a variedade Catuaí Vermelho, nos estádios de maturação verde, verdoengo e cereja. A partir dos resultados verificou-se que o módulo de deformidade reduz na medida em que os frutos passam do estádio de maturação verde para cereja.

Visando entender os esforços necessários para desprendimentos dos frutos de café, Silva et al. (2010) desenvolveram um dinamômetro portátil o qual possibilitou a realização dos ensaios diretamente na planta. Experimentos foram realizados em diversas variedades, nos estádios de maturação verde, cereja, passa e seco, em cinco épocas numa mesma colheita. Foram observadas diferenças significativas nas forças de desprendimento dos frutos entre os estádios de maturação obtendo-se valores superiores para o estádio de maturação verde.

Diante do exposto objetivou-se, com este trabalho, determinar e avaliar as propriedades geométricas, físicas e mecânicas do sistema fruto-pedúnculo-ramo do cafeeiro, tal como avaliar os efeitos do estádio de maturação sobre essas propriedades.

\section{Material e Métodos}

A pesquisa foi executada utilizando-se amostras de café arábica, variedade Catuaí Vermelho IAC 144, coletadas no período maio/2013 a julho/2013. As amostras foram coletadas sempre pela manhã e os ensaios realizados durante o mesmo dia da coleta. Para esta coleta foram escolhidas plantas aleatórias de uma área experimental da Universidade Federal de Viçosa, em Viçosa, MG, sendo as amostras coletadas aleatoriamente, ao longo da planta.

Foram determinadas as dimensões, massa, volume, massa específica, módulo de elasticidade, coeficiente de Poisson e razão de amortecimento para o sistema fruto-pedúnculo e para o ramo. Foram considerados, especificamente para o sistema fruto-pedúnculo, os estádios de maturação verde e cereja.

Para determinação das dimensões, módulo de elasticidade e coeficiente de Poisson dos frutos, pedúnculos e ramos, foi empregada uma câmera digital Samsung PL 120 com resolução de 14 megapixels, cujos vídeos e imagens foram processados posteriormente, por meio do programa computacional de código aberto Scilab, versão 5.4.

Para cada estádio de maturação foram capturadas imagens de 120 amostras do sistema fruto-pedúnculo sobre uma malha graduada em milímetros; de forma similar, outras 120 imagens de amostras foram adquiridas para o ramo plagiotrópico.

As imagens capturadas foram processadas a fim de determinar o comprimento e o diâmetro equatorial do fruto e o comprimento e o diâmetro do pedúnculo, além do diâmetro médio do ramo.

As massas médias dos frutos e pedúnculos foram determinadas a partir de 120 amostras para cada estádio de maturação utilizando-se uma balança digital com precisão 0,01 g. As massas dos frutos foram mensuradas individualmente enquanto que para os pedúnculos se usaram conjuntos de dez 
amostras. A massa média dos ramos foi obtida a partir de 120 amostras, mensuradas em conjuntos de dez amostras.

O volume médio de 120 amostras dos frutos em cada estádio de maturação e do ramo foi mensurado utilizandose uma proveta de $100 \mathrm{~mL}$ com precisão de $1,0 \mathrm{~mL}$. Para o pedúnculo, 120 amostras em cada estádio de maturação, utilizou-se uma proveta de $10 \mathrm{~mL}$ com precisão de $0,1 \mathrm{~mL}$. Para os frutos, pedúnculos e ramos, as medições dos volumes foram realizadas imergindo um conjunto de dez amostras em água, conforme proposto por Santos (2008).

A massa específica dos frutos, pedúnculo e ramos, foi determinada por meio da razão entre os valores de massa e volume obtidos experimentalmente.

Os módulos de elasticidade dos frutos, pedúnculos e ramos, foram determinados por meio de ensaios mecânicos de compressão, tração e flexão, respectivamente. As forças foram providas por um atuador hidráulico linear com velocidade de $1,0 \mathrm{~mm} \mathrm{~s}^{-1} \mathrm{e}$ monitoradas por meio de célula de carga, modelo LC 101 fabricado por Omega LC 101 com capacidade $100 \mathrm{lbf}$, conectada a um sistema de aquisição de dados HBM, modelo Spider 8, gerenciado por computador pelo software HBM Catman 2.2 (liçenca: AMOTDP\#RXXX). Os deslocamentos da placa móvel foram capturados em vídeos e posteriormente processados para obtenção das deformações.

O módulo de elasticidade dos frutos foi calculado a partir do método de Hertz (Mohsenin et al., 1963), o qual é utilizado para determinação do módulo de elasticidade de corpos convexos comprimidos entre duas placas planas paralelas, conforme metodologia empregada por Couto et al. (2002) e Ribeiro et al. (2007).

O módulo de elasticidade foi calculado utilizando-se valor de força de compressão e deformação elástica correspondente a uma deformação específica de $0,01 \mathrm{~m} \mathrm{~m}^{-1}$. O ensaio foi realizado em 12 amostras do fruto para cada um dos estádios de maturação.

Previamente aos ensaios de tração, imagens das amostras do pedúnculo sobre malha graduada em milímetros foram capturadas através de uma câmera digital Samsung PL 120, para determinação do comprimento e diâmetro do pedúnculo.

A partir dos dados de tensão em função da deformação específica, o módulo de elasticidade foi calculado por meio da tangente à curva de tensão-deformação na região elástica, sendo o pedúnculo tratado como material homogêneo e isotrópico. O ensaio foi empregado em 12 amostras do pedúnculo para cada estádio de maturação.

O módulo de elasticidade dos ramos foi determinado empregando-se amostras de ramos com $250 \mathrm{~mm}$ de comprimento sendo biapoiadas nas extremidades e submetidas a carregamento na região central.

O módulo de elasticidade de flexão foi calculado pela Eq. 1 .

$$
\mathrm{E}=\frac{\mathrm{Fl}^{3}}{48 \mathrm{I} \Delta \mathrm{l}}
$$

$$
\begin{array}{cl}
\text { em que: } & \\
\text { E } & \text { - módulo de elasticidade, } \mathrm{Pa} \\
\mathrm{F} & \text { - força de flexão, } \mathrm{N} \\
\mathrm{I} & \text { - momento de inércia do ramo, } \mathrm{m}^{4} \\
\mathrm{l} & \text { - comprimento do vão de flexão, m }
\end{array}
$$

\section{$\Delta \mathrm{l}$ - flecha de deformação do ramo, m}

Previamente aos ensaios, imagens das amostras dos ramos sobre malha graduada em milímetros foram capturadas para determinação do diâmetro médio necessário para cálculo do momento de inércia. $\mathrm{O}$ módulo de elasticidade foi determinado utilizando-se valor de flecha de deformação de $10 \mathrm{~mm}$ e a força de flexão correspondente, considerando-se 12 amostras do ramo.

Os coeficientes de Poisson para os frutos e pedúnculos foram determinados considerando-se 12 amostras do fruto e 12 do pedúnculo, em cada estádio de maturação. As amostras de fruto e pedúnculo foram submetidas à compressão e tração respectivamente, por meio de um atuador hidráulico linear. Imagens das amostras antes e durante o ensaio, foram capturadas e posteriormente processadas para determinação das deformações transversais e longitudinais. O coeficiente de Poisson foi determinado a partir da razão entre a deformação transversal e a longitudinal, conforme proposto por Rao (1995).

Amostras dos sistemas fruto-pedúnculo e de ramos com comprimento $300 \mathrm{~mm}$ foram excitadas utilizando-se um sistema produzido pela LDS (Ling Dynamic Systems) composto por um gerador de sinais COMET USB da marca Dactron, um amplificador LDS PA100E-CE e uma máquina vibradora eletromagnética modelo V-406. O gerador de sinais foi controlado por computador por meio de programa computacional fornecido pelo fabricante. As amostras foram presas ao sistema de fixação por placas que, por sua vez, foram fixadas à máquina vibradora eletromagnética.

Excitações do tipo função impulso foram geradas pela máquina vibradora eletromagnética e usadas para induzir os deslocamentos nas amostras. Tais deslocamentos foram capturados por uma câmera de alta velocidade Mega Speed HHC modelo X3, com capacidade de captura de 1000 quadros por segundo. As imagens foram processadas em software Scilab, versão 5.4, obtendo-se o deslocamento de um ponto da amostra em função do tempo. Por meio da curva de decaimento e se utilizando método de decremento logaritmo, a razão de amortecimento foi calculada conforme Rao (1995). A razão de amortecimento foi determinada para 10 amostras do sistema fruto-pedúnculo em cada estádio de maturação e para 10 amostras do ramo.

Os dados foram submetidos à análise de variância, segundo delineamento inteiramente casualizado, com dois tratamentos (estádios de maturação verde e cereja). O efeito dos estádios de maturação sobre o comportamento das propriedades geométricas, físicas e mecânicas foi estudado por meio do teste de Tukey, a nível de significância de 0,05 . As análises estatísticas foram realizadas por meio do programa computacional $\mathrm{R}$, versão 2.15 .

\section{Resultados e Discussão}

As dimensões dos frutos, comprimento e diâmetro, aumentaram na medida em que se passou do estádio de maturação verde para o cereja (Tabela 1). Este comportamento, observado por Ciro (2001), Yung \& Fridley (1974) e Rodríguez et al. (2006), pode ser explicado pelo desenvolvimento do endosperma e perisperma, por meio de divisão e alongamento celular (Castro \& Marraccini, 2006). 
O diâmetro do pedúnculo aumentou sempre que o estádio de maturação (Tabela l) evoluiu, concordando com o encontrado por Yung \& Fridley (1974), Ciro (2001) e Rodríguez et al. (2006). Já para o comprimento do pedúnculo diferenças significativas não foram encontradas entre os estádios de maturação empregando-se o teste Tukey a nível de 0,05 de significância.

Para o ramo obteve-se diâmetro médio de 5,06 mm, com desvio padrão $0,85 \mathrm{~mm}$.

A massa e o volume do fruto aumentaram na medida em que se passou do estádio de maturação verde para o cereja (Tabela 2), conforme reportado por Ciro (2001), Rodríguez et al. (2006) e Santos (2008). Este comportamento também pode ser explicado pela divisão e alongamento das células dos frutos ocasionando aumentos de massa e volume (Castro \& Marraccini, 2006).

A massa específica do fruto reduziu na medida em que o estádio de maturação (Tabela 2 ) reduziu em concordância com o obtido por Santos (2008) enquanto Ciro (2001) e Rodríguez et al. (2006), obtiveram tendência de aumento na massa específica porém em magnitudes desprezíveis. Tal comportamento está relacionado com as taxas nas quais os frutos aumentaram seus volumes e suas massas. Verificou-se, portanto, no presente trabalho, uma taxa de aumento do volume superior à taxa de aumento da massa ocasionando redução da massa específica do fruto.

Para o pedúnculo, a massa, o volume e a massa específica em ambos os estádios de maturação foram estatisticamente iguais a nível de 0,05 de significância pelo teste Tukey.

Obteve-se, para o ramo, massa específica média $0,90 \mathrm{~g}$ $\mathrm{cm}^{-3}$ com desvio-padrão $0,11 \mathrm{~g} \mathrm{~cm}^{-3}$. Filgueiras (2001) obteve $0,978 \mathrm{~g} \mathrm{~cm}^{-3}$ para ramos de $5,4 \mathrm{~mm}$. O valor inferior ao encontrado no presente trabalho pode ser devido às variações nas propriedades físicas em função da espécie, variedade, idade e tipo de manejo do cafeeiro, além de condições climáticas (Aristizábal et al., 2003; Rodríguez et al., 2006).

Para os frutos o módulo de elasticidade reduziu na medida em que evoluiu do estádio de maturação verde para o cereja (Tabela 3). Esta redução, também observada por Couto et al. (2002), é devida à redução da rigidez da mucilagem, a qual sofre um processo de desestruturação na medida em que ocorre

Tabela 1. Valores médios das propriedades geométricas para o fruto e pedúnculo, nos estádios de maturação verde e cereja

\begin{tabular}{|c|c|c|c|c|}
\hline \multirow{3}{*}{$\begin{array}{c}\text { Estádio } \\
\text { de maturação }\end{array}$} & \multicolumn{2}{|c|}{ Fruto } & \multicolumn{2}{|c|}{ Pedúnculo } \\
\hline & Com & Diâ & Com & Diâ \\
\hline & \multicolumn{4}{|c|}{$(\mathrm{mm})$} \\
\hline Verde & $16,14 \mathrm{~A}$ & $12,77 \mathrm{~A}$ & $6.64 \mathrm{~A}$ & $2,12 \mathrm{~A}$ \\
\hline Cereja & $17,12 \mathrm{~B}$ & $14,76 \mathrm{~B}$ & $6,36 \mathrm{~A}$ & $2,32 \mathrm{~B}$ \\
\hline
\end{tabular}

As médias seguidas de pelo menos uma mesma letra não diferem estatisticamente entre si, pelo teste de Tukey a nível de $5 \%$ de probabilidade

Com - Comprimento, Dia - Diãmetro a maturação do fruto adquirindo a forma de gel (Castro \& Marraccini, 2006). Associado à desestruturação da mucilagem, ocorre também o enfraquecimento das paredes celulares do fruto favorecido pela ação de enzimas (Trevisan, 2012).

O módulo de elasticidade do pedúnculo e a força de desprendimento do fruto também reduziram na medida em que evolui do estádio de maturação verde para o cereja (Tabela 3). Este comportamento, observado por diversos autores (Yung \& Fridley, 1974; Sampaio, 2000; Ciro, 2001; Rodríguez et al., 2006; Silva et al., 2010), se deve ao fato de ocorrer maior ligação entre o fruto, o pedúnculo e a planta no estádio de maturação verde, para melhor condução e fornecimento de nutrientes ao fruto, a fim de promover seu desenvolvimento. Além disto, ocorre degradação da parede celular do pedúnculo em virtude da ação de enzimas ativadas pelo hormônio etileno. A degradação da parede celular torna o pedúnculo frágil reduzindo seu módulo de elasticidade e a força necessária para seu rompimento (Trevisan, 2012).

Em experimento no qual foram utilizadas amostras de café da variedade Catuaí Vermelho, Couto et al. (2002) obtiveram valores próximos a 9,0 e 45,0 MPa para módulo de elasticidade de frutos cereja e verde, respectivamente. As diferenças de magnitudes podem estar relacionadas às variações das propriedades mecânicas em função da espécie, idade, manejo da planta, entre outros (Aristilzábal et al., 2003). Além disto, também podem estar relacionadas a parâmetros do ensaio, como a velocidade de compressão e a deformação específica empregada.

Rodríguez et al. (2006) determinaram módulos de elasticidade com magnitudes 15,88 e 4,73 MPa para pedúnculos do cafeeiro da variedade Catuaí nos estádios cereja e verde, respectivamente. Ciro (2001) obteve valores de 22,61 e 23,14 $\mathrm{MPa}$, para pedúnculo nos estádios de maturação cereja e verde, respectivamente para café variedade Colombiano. Santos (2008) utilizou, para modelar o comportamento dinâmico do sistema fruto-pedúnculo, valores 26,06 e 31,03 MPa, para o pedúnculo nos estádios cereja e cereja, respectivamente, obtidos por Yung \& Fridley (1974).

Os módulos de elasticidade obtidos no presente trabalho apresentam magnitudes inferiores às encontradas por Yung \& Fridley (1974), Ciro (2001) e Rodríguez et al. (2006). Uma

Tabela 3. Valores médios para módulo de elasticidade do fruto e pedúnculo e força de desprendimento do fruto, em ambos os estádios de maturação

\begin{tabular}{cccc}
\hline Estádio & MEF & MEP & FDF \\
\cline { 2 - 3 } de maturação & \multicolumn{1}{c}{ (N) } \\
Verde & (MPa) & & $8,37 \mathrm{~A}$ \\
Cereja & $2,93 \mathrm{~A}$ & $3,91 \mathrm{~A}$ & \\
\hline
\end{tabular}

As médias seguidas de pelo menos uma mesma letra não diferem estatisticamente entre si, pelo teste de Tukey a nível de 0,05 de probabilidade

MEF - Módulo de elasticidade do fruto, MEP - Módulo de elasticidade do pedúnculo, FDF Força de desprendimento do fruto

Tabela 2. Valores médios de massa, volume e massa específica do fruto e pedúnculo, nos estádios de maturação verde e cereja

\begin{tabular}{|c|c|c|c|c|c|c|}
\hline \multirow{2}{*}{$\begin{array}{c}\text { Estádio } \\
\text { de maturação }\end{array}$} & \multicolumn{3}{|c|}{ Fruto } & \multicolumn{3}{|c|}{ Pedúnculo } \\
\hline & Massa (g) & Volume $\left(\mathrm{cm}^{3}\right)$ & Massa especifica $\left(\mathrm{g} \mathrm{cm}^{-3}\right)$ & Massa $(\mathrm{g})$ & Volume $\left(\mathrm{cm}^{3}\right)$ & Massa especifica $\left(\mathrm{g} \mathrm{cm}^{-3}\right)$ \\
\hline Verde & $1,04 \mathrm{~A}$ & $0,92 \mathrm{~A}$ & $1,13 \mathrm{~A}$ & $0,03 \mathrm{~A}$ & $0,03 \mathrm{~A}$ & $1,09 \mathrm{~A}$ \\
\hline Cereja & $1,38 \mathrm{~B}$ & $1,37 \mathrm{C}$ & $1,02 \mathrm{~B}$ & $0,03 \mathrm{~A}$ & $0,02 \mathrm{~A}$ & $1,46 \mathrm{~A}$ \\
\hline
\end{tabular}

As médias seguidas de pelo menos uma mesma letra não diferem estatisticamente entre si, pelo teste de Tukey a nível de 0,05 de probabilidade 
provável explicação é a variação das propriedades geométricas e mecânicas de uma estrutura biológica, no caso o pedúnculo, em função da espécie, variedade, condição climática, idade da planta ou até mesmo do tipo de manejo realizado (Aristizábal et al., 2003; Rodríguez et al., 2006). Por outro lado, as diferenças também podem estar relacionadas com o tipo de ensaio já que Ciro (2001) e Rodríguez et al. (2006) determinaram o módulo de elasticidade por meio de ensaio de flexão, considerando o sistema fruto-pedúnculo com uma viga engastada em umas extremidades.

Sampaio (2000) encontrou, para café variedade Catuaí Vermelho, forças de desprendimento entre 6,8 a 9,87 e 3,17 a $3,63 \mathrm{~N}$, para estádio de maturação verde e cereja, respectivamente enquanto Silva et al. (2010), obtiveram força de desprendimento entre 7,54 a 11,81 e 5,44 a $7,21 \mathrm{~N}$, para estádio de maturação verde e cereja, respectivamente, para café Catuaí vermelho. Os resultados obtidos no presente trabalho para força de desprendimento do fruto apresentam valores próximos ou até mesmo dentro dos intervalos de valores encontrados por Sampaio (2000) e Silva et al. (2010).

Para o ramo obteve-se o módulo de elasticidade com valor médio 1,940 GPa e desvio padrão 0,622 GPa. Aristizábal et al. (2003) encontraram valores entre 2,26 e 8,43 GPa para os ramos de café das variedades Vermelho Caturra, Colômbia Vermelho e Colômbia Amarelo. Filgueiras (2001) obteve 3,56 GPa para ramos de 5,4 mm de diâmetro. O menor valor para módulo de elasticidade do ramo pode estar relacionado à sua menor massa específica, $0,978 \mathrm{~g} \mathrm{~cm}^{-3}$ obtida por Filgueiras (2001) comparada com $0,900 \mathrm{~g} \mathrm{~cm}^{-3}$ obtido no presente trabalho. Além disto, podem ocorrer variações nas propriedades mecânicas do ramo em função da variedade, espécie, condições climáticas, idade, tipo de manejo da planta do cafeeiro (Aristizábal et al., 2003; Rodríguez et al., 2006).

Não foram encontradas diferenças significativas entre os valores de coeficiente de Poisson para o fruto e o pedúnculo nos dois estádios de maturação (Tabela 4) porém o fruto apresentou tendência de maior coeficiente de Poisson no estádio de maturação cereja em relação ao estádio verde significando que, para determinada deformação longitudinal, os frutos cereja apresentam uma deformação maior na direção longitudinal quando comparados aos frutos verdes. Tal comportamento pode estar relacionado à desestruturação da mucilagem e a degradação da parece celular do fruto-pedúnculo favorecendo as deformações transversais (Castro \& Marraccini, 2006)

Rodríguez et al. (2006) obtiveram valores de 0,3191 e 0,3457 para pedúnculos cereja e verdes, respectivamente, de plantas de café variedade Catuaí. Comparando os resultados obtidos pelos autores com os obtidos no presente trabalho, verificam-se resultados com magnitudes próximas, principalmente para o estádio de maturação cereja.

Tabela 4. Valores médios para coeficiente de Poisson do fruto e pedúnculo nos estádios de maturação verde e cereja

\begin{tabular}{ccc}
\hline Estádio de maturação & \multicolumn{2}{c}{ Coeficiente de Poisson } \\
\cline { 2 - 3 } Verde & Fruto & Pedúnculo \\
Cereja & $0,24 \mathrm{~A}$ & $0,35 \mathrm{~A}$ \\
\hline
\end{tabular}

As médias seguidas de pelo menos uma mesma letra não diferem estatisticamente entre si, pelo teste de Tukey a nível de 0,05 de probabilidade
Obteve-se 0,15 e 0,09 para a razão de amortecimento do pedúnculo nos estádios de maturação verde e cereja, respectivamente, determinadas por meio das curvas de decaimento (Figura 1). Para o ramo encontrou-se razão de amortecimento com valor médio 0,02 e desvio padrão 0,01 .

Ao comparar os valores para os estádios de maturação, diferenças significativas não foram encontradas a nível de $5 \%$ pelo teste Tukey. Analisando a tendência dos valores, a razão
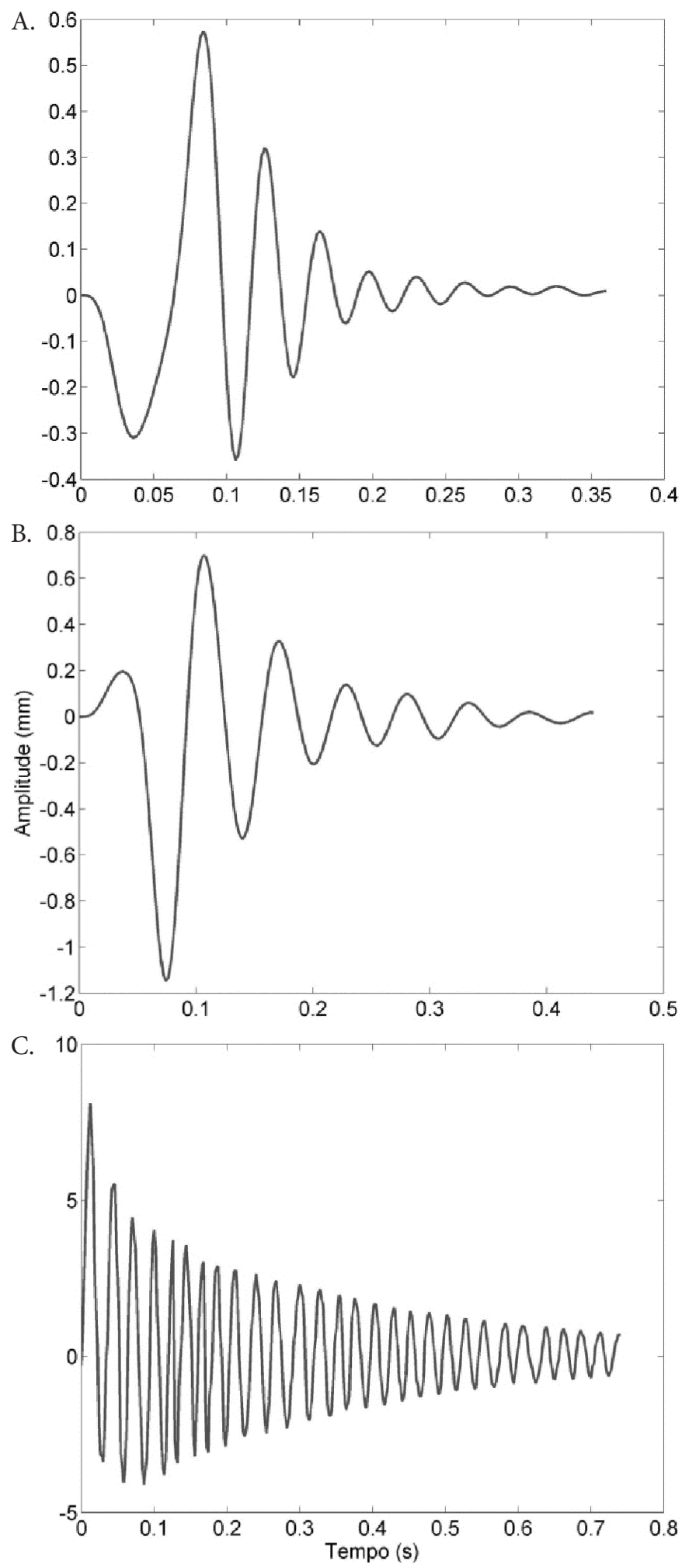

Figura 1. Curva de decaimento obtida experimentalmente, pedúnculo nos estádios de maturação cereja $(A)$, verde $(B)$ e para o ramo $(\mathrm{C})$ 
de amortecimento do pedúnculo reduziu na medida em que se passa do estádio de maturação verde para o cereja, significando que o sistema fruto-pedúnculo no estádio de maturação verde apresentou maior capacidade de amortecimento da excitação à qual foi submetido.

Aristilzábal et al. (2003) encontraram, também utilizando método de decremento logaritmo, valores entre 0,04 e 0,10 para razão de amortecimento do sistema tronco-ramo do cafeeiro; para o sistema tronco-ramo-folhas os autores obtiveram valores entre 0,08 e 0,1 sinalizando que as folhas do cafeeiro favorecem o amortecimento de excitações provocadas na planta.

Os valores para razão de amortecimento obtidos no presente trabalho e por Aristilzábal et al. (2003) são menores que 1,0, caracterizando a planta do cafeeiro e suas partes como sistema subamortecido (Rao, 1995).

\section{Conclusões}

1. Existe tendência de aumento nas dimensões, na massa e no volume dos frutos na medida em que o estádio de maturação evoluiu enquanto para a massa específica há tendência de redução na medida em que o fruto evolui seu estádio de maturação.

2. Os módulos de elasticidade para o pedúnculo e o fruto tendem a reduzir na medida em que seu estádio de maturação evolui. Maiores magnitudes de forças foram necessárias para desprendimento dos frutos verdes quando comparados com os frutos cerejas.

3. Os valores obtidos para a razão de amortecimento dos pedúnculos e dos ramos foram menores que o unitário caracterizando o sistema fruto-pedúnculo e ramo do cafeeiro como subamortecido.

\section{Agradecimentos}

Os autores agradecem ao Conselho Nacional de Desenvolvimento Cientifico e Tecnológico $(\mathrm{CNPq})$ e à Fundação de Amparo à Pesquisa do Estado de Minas Gerais (FAPEMIG) pelo auxílio financeiro em prol do desenvolvimento da pesquisa.

\section{Literatura Citada}

Albuquerque, E. L. Investigaçaõ sobre a variabilidade da resposta dinâmica de painéis reforçados.Campinas: UNICAMP, 2005. 72p. Dissertação Mestrado

Aristzábal, I. D.; Oliveros, T. C. E.; Alvares, M. F. Mechanical harvest of coffee applying circular and multidirectional vibrations. Transactions of the ASAE, v.46, p.205-209, 2003.

Barbosa, J. A.; Salvador, N.; Silva, F. M. Desempenho operacional de derriçadoras mecânicas portáteis, em diferentes condições de lavouras cafeeiras. Revista Brasileira de Engenharia Agrícola e Ambiental, v.9, p.129-132, 2005. http://dx.doi.org/10.1590/ S1415-43662005000100020

Castro, R. D.; Marraccini, P. Citology, biochemistry and molecular changes during coffee fruit development. Brazilian Journal of Plant Physiology, v.18, p.175-199, 2006. http://dx.doi.org/10.1590/ S1677-04202006000100013
Ciro, H. J. Coffee harvesting I: Determination of the natural frequencies of the fruit stem system in coffee tress. Applied Engineering in Agriculture, v.17, p.475-479, 2001.

CONAB - Companhia Nacional de Abastecimento. Acompanhamento da safra brasileira: CaféSafra 2013 Segunda Estimativa, Maio/2013.< http://www.conab.gov.br/conteudos.php?a=1253\&t>. 31 Jul. 2013.

Couto, S. M; Batista, C. S; Peixoto, A. B; Devilla, I. A. Comportamento mecânico dos frutos de café: módulo de deformidade. Revista Brasileira de Engenharia Agrícola e Ambiental, v.6, p.285-294, 2002. http://dx.doi.org/10.1590/S1415-43662002000200018

Erdogan, D.; Guner, M; Dursun, E.; Gezer, I. Mechanical Harvesting of Apricots. Biosystems Engineering, v.85, p.19-28, 2003. http:// dx.doi.org/10.1016/S1537-5110(03)00024-2

Filgueiras, W. H. Modelagem da planta de café por elementos finitos para estudos de colheita por vibração. Viçosa: UFV, 2001. 81p. Dissertação Mestrado

Mohsenin, N. N.; Cooper, H. E.; Tukey, L. D. Engineering approach to evaluation of textural factor in fruits and vegetables, Transactions of the ASAE, v.6, p.85-92, 1963. http://dx.doi. org/10.13031/2013.40832

Pezzi, F.; Caprara, C. Mechanical grape harvesting: Investigation of the transmission of vibrations. Biosystems Engineering, v.103, p.281286, 2009. http://dx.doi.org/10.1016/j.biosystemseng.2009.04.002

Polat, R.; Gezer, I.; Guner, M.; Dursun, E.; Erdogan, D.; Bilim, H. C. Mechanical harvesting of pistachio nuts. Journal of Food Engineering, v.79, p.1131-1135, 2007. http://dx.doi.org/10.1016/j. jfoodeng.2006.03.023

Rao, S. S. Mechanical vibrations. 3.ed. New York: Addison-Wesley Publishing Company, 1995. 912p.

Ribeiro, D. M.; Corrêa, P. C.; Furtado, B. F.; Goneli, A. L. D.; Resende, O. Propriedades mecânicas dos grãos de soja em função do teor de água. Engenharia Agrícola, v.27, p.493-500, 2007. http://dx.doi. org/10.1590/S0100-69162007000300019

Rodríguez, A. M.; Queiroz, D. M.; Espinosa, B. G.; Zandonadi, R. Determinácion de propriedades físico-mecánicas de los frutos de café (Coffea arabica variedade Catuai) relacionadas con la cosecha mecanizada. Revista Ciências Técnicas Agropecuárias, v.15, p.22-27, 2006.

Sampaio, C. P. Determinação da força requerida para o desprendimento de frutos de café em diferentes estádios de maturação. Viçosa: UFV, 2000. 48p. Dissertação Mestrado

Sanders, K. F. Orange harvesting systems review. Biosystems Engineering, v.90, p.115-125, 2005. http://dx.doi.org/10.1016/j. biosystemseng.2004.10.006

Santos, F. L. Simulação e avaliação do comportamento dinâmico de frutos do cafeeiro na derriça. Viçosa: UFV, 2008. 136p. Tese Doutorado

Santos, F. L.; Queiroz, D. M. de; Pinto, F. A. C.; Resende, R. C. Efeito da frequência e amplitude de vibração sobre a derriça de frutos de café. Revista Brasileira de Engenharia Agrícola e Ambiental, v.14, p.425-431, 2010a. http://dx.doi.org/10.1590/ S1415-43662010000400012

Santos, F. L.; Queiroz, D. M. de; Pinto, F. A. C.; Santos, N. T. Analysis of the coffee harvesting process using an electromagnetic shaker. Acta Scientiarum. Agronomy, v. 32, p.373-378, 2010 b.

Sessiz, A.; Ozcan, M. T. Olive removal with pneumatic branch shaker and abscission chemical. Journal of Food Engineering. v.76, p.148-153, 2006. http://dx.doi.org/10.1016/j.jfoodeng.2005.05.002 
Silva, F.C.; Silva, F. M.; Alves, M. C; Barros, M.M.; Sales, R.S. Comportamento da força de desprendimento dos frutos de cafeeiro ao longo do período de colheita. Revistas Ciência e Agrotecnologia, v.34, p.468-474, 2010. http://dx.doi.org/10.1590/ S1413-70542010000200028

Souza, C. M. A; Queiroz, D. M. de; Rafull, L. Z. D. Derriçadora portátil na colheita total e seletiva de frutos do cafeeiro. Pesquisa Agropecuária Brasileira, v.41, p.1637-1642, 2006. http://dx.doi. org/10.1590/S0100-204X2006001100010
Taplak, H.; Parlak, M. Evaluation of gas turbine rotor dynamic analysis using the finite element method. Measurement, v.45, p.1089-1097, 2012. http://dx.doi.org/10.1016/j.measurement. 2012.01.032

Trevisan, M. J. Efeito da aplicação de 1-metilciclopropeno e etileno na fisiologia e no amadurecimento de mamões “Golden”. Piracicaba: USP, 2012. 116p. Tese Doutorado

Yung, C.; Fridley, R. B. Computer analysis of fruit detachment during tree shaking. St. Joseph: ASAE, 1974. p.48-60. Paper n.74-3009. 